\title{
Sequential effects in auditory choice reaction time tasks
}

\author{
PHILIP T. QUINLAN \\ University of York, York, England
}

\begin{abstract}
This paper concerns sequential effects in choice reaction time tasks. Performance in two interleaved auditory tasks was examined, and two general types of sequential effects were revealed. First, a response repetition effect occurred: Subjects were facilitated in responding when both the stimulus and the response were immediately repeated. Generally, it appeared that subjects were operating according to the bypass rule-that is, repeat the response if the stimulus or some aspect thereof is repeated from the preceding trial; otherwise, change the response. In addition, the experiment also revealed a second type of sequential effect, known as a task-switching effect. Subjects were overall slower to respond when the task changed between adjacent trials than when there was no task change. A final result was that subjects were markedly impaired when the stimulus changed but the same response had to be repeated. This finding has been reported elsewhere when purely visual tasks have been used. Hence, it seems that particular difficulties arise, in such sequential testing situations, when type-distinct stimuli are grouped into the same response categories.
\end{abstract}

This paper reports on a study that concerns sequential effects in choice reaction time (CRT) tasks. Although there was much interest in this topic more than 20 years ago, the present research extends some recent work of Quinlan and Hill (in press). In that study, Quinlan and Hill were particularly interested in two basic effects: (1) the repetition effect (Bertelson, 1965; Rabbitt, 1992) and (2) the modality-shifting effect (Spence \& Driver, 1997). Central to both effects is how performance on trial $n+1$ may be influenced by what transpired on trial $n$. For expository convenience, the first trial of any such pair (trial $n$ ) will be referred to as the prime trial, and the second member of each pair will be referred to as the target trial (trial $n+1)$. According to Rabbitt (1992, p. 313), the repetition effect refers to the finding that "CRTs are significantly faster when the signals and responses that occur on successive trials are identical ... than when they are different." In contrast, the modality-switching effect is that reaction times (RTs) to targets are faster when the target and prime appear in the same modality on adjacent trials (ipsimodal trials) than when the target and prime appear in different modalities (crossmodal trials; see Spence \& Driver, 1997).

Evidence for both effects was reported by Quinlan and Hill (in press). In their study, the subjects were given two tasks - a visual task and an auditory task - to perform in each experiment. Only one task was carried out on each trial, but the two tasks were intermixed within a block of trials. Each task demanded a binary judgment about the

This research has benefited from discussions with Nick Hill and Steven Roodenrys. Correspondence concerning this article should be addressed to P. Quinlan, Department of Psychology, University of York, Heslington, York YO10 5DD, England (e-mail: ptql@york.ac.uk). left/right location of an imperative signal, and the subjects responded by pressing one of two finger keys. In the auditory task, subjects made a positional judgment about a monaural tone presented over headphones. In the visual task, the subjects made the same positional judgment about a luminance increment in a $3 \times 3$ array of boxes presented on the screen of a visual display unit.

In the first two experiments and on each trial, a preparatory signal was presented prior to each imperative signal, to indicate the relevant stimulus modality. At a short stimulus onset asynchrony (SOA) between the preparatory and the imperative signal (i.e., $60 \mathrm{msec}$ ), the subjects were quicker to repeat the same response than to change their response when presented with successive tones. No such repetition effect occurred on the visual target trials. The pattern of performance on the auditory target trials was interpreted in terms of what has been termed the bypass rule (Fletcher \& Rabbitt, 1978; Krueger \& Shapiro, 1981). According to the bypass rule, subjects are predisposed to repeat the same response if the stimuli are the same across adjacent trials, and they will tend to change their response if the stimuli differ. The lack of a repetition effect on the visual target trials was argued to be due to the subjects having a general bias to attend to the visual modality; consequently, they essentially were able to overcome any deleterious effects of having to change their response across adjacent trials.

A modality-switching effect was also present in the data, because subjects were impaired if the stimulus modality changed across successive trials, regardless of the modality of the target. Indeed, Quinlan and Hill (in press) went on to argue that the repetition effect and the modality-switching effect are essentially independent. When the SOA was increased (i.e., to $500 \mathrm{msec}$ ) and the preparatory signals were omitted, the modality-switching 
costs were still evident, but the repetition effect was abolished. Indeed, under these circumstances, the subjects were generally inhibited in repeating a response if the target appeared at the same location as the prime. That is, an inhibition of return effect (Posner \& Cohen, 1984; Terry, Valdes, \& Neill, 1994) occurred and was present on both the auditory and the visual target trials (cf. ReuterLorenz, Jha, \& Rosenquist, 1996).

Quinlan and Hill (in press) concluded that the repetition effect and modality-switching effect reflect different underlying processes. They argued that, whereas the repetition effect may well reflect compliance with the bypass rule (Fletcher \& Rabbitt, 1978; Krueger \& Shapiro, 1981), the modality-switching effect appears to reflect costs in having to switch attention between the auditory and the visual modalities. In addition, the presence of inhibition of return in the final experiment seemed to be more closely related to some form of spatial-orienting mechanism. The pattern of performance across the various experiments also supported the view that spatial orienting and modality switching reflect the operation of quite different attentional mechanisms.

Prior to the study by Quinlan and Hill (in press), nearly all of the work on sequential effects in CRTs had been carried out in the visual domain. For instance, Pashler and Baylis (1991) reported a study in which subjects classified visually presented keyboard characters according to various classification schemes. For example, in a particular experiment, the subjects were presented on each trial with one of six possible characters and had to use the tertiary classification scheme: " 2 " and "4," key one; "P" and "V," key two; "\&" and "\#," key three (see, also, Campbell \& Proctor, 1993). Rogers and Monsell (1995) examined performance in similar classification tasks in which subjects were presented with alphanumeric characters. Allport, Styles, and Hsieh (1994) examined performance in a range of tasks, but in all of them, the stimuli were presented visually. Under these conditions, the costs of switching between tasks had been examined in the absence of any change in the input modality. In contrast, the study by Quinlan and Hill examined the nature of sequential effects when the same task was carried out in different modalities. Now, an aim was to examine what possible costs might be associated with having to switch between different tasks when all the stimuli were presented in the auditory modality. Under these conditions, the notion of a modality-switching effect is irrelevant, as both tasks demand attention to the auditory modality. On these grounds, therefore, any costs associated with switching tasks across trials presumably reflect something Rogers and Monsell (1995) have termed task-set reconfiguration (p. 207). According to Rogers and Monsell, "to adopt a task-set is to select, link, and configure the elements of a chain of processes that will accomplish a task" $(1995$, p. 208). Task-set reconfiguration, therefore, refers to the ability to discard one chain of processes and invoke another.
An aim of the present paper was, therefore, to examine the nature of sequential effects in auditory CRT tasks. Little is known about the control processes that operate within the auditory domain (although, see Scharf, 1998, for a review), and it was hoped that the present study would throw further light on these. In addition, it was of interest to see the degree to which performance on the auditory tasks corresponded to that found when purely visual tasks are used.

In an earlier pilot work, Quinlan and Hill had examined performance under circumstances in which, in a visual task, subjects made a position judgment about the location of a luminance increment and, in an auditory task, made a binary pitch judgment (i.e., high vs. low pitch) about a binaural tone. Generally, the data from this study were in agreement with those of the experiment in which subjects made the same locational decisions about the auditory and visual targets (Quinlan \& Hill, in press). The simple idea here, therefore, was to combine the pitch judgment with the auditory positional judgment. So, in the present experiment, on half the trials the subjects made high/low pitch judgments about a binaural target tone, and on the remaining half of the trials, they made left/ right positional judgments about a monaural target tone. From the previous studies, there was some evidence to suggest that subjects find the pitch judgments more difficult to perform than the positional judgments. Mean RTs for the pitch judgments were around $80 \mathrm{msec}$ slower than comparable mean RTs for the position judgments. Such a difference is in accord with the data reported by BenArtzi and Marks (1995). They examined performance in binary pitch judgment tasks similar to those used here, together with performance on a visual spatial task. In the visual task, the subjects were presented on the screen of a computer with a dot that was located either above or below the horizontal midline. The positional judgment in this case was high or low, depending on the location of the dot. Various differences in pitch were combined across different blocks of trials. In addition, the spatial separation between the two dot positions was also varied. For all cases, the subjects found the positional judgment easier to make than the pitch judgment; moreover, the difficulty of pitch judgment was clearly dependent on the frequency separation of the two tones used. When there was only an $80-\mathrm{Hz}$ difference between the tones, the subjects' performance was considerably impaired, relative both to the other pitch judgments and to the visual position judgment. In the present experiment, the pitch separation between the two tone targets was only $35 \mathrm{~Hz}$, in order to maintain parity with Quinlan and Hill (in press). Therefore, it seems reasonable to assume that the present pitch judgment is relatively difficult.

Apart from the inherent difficulty of the respective judgments, there are other task constraints that should be taken into account. These relate to the different stimulusresponse compatibilities across the two tasks. When making the positional judgments, the subjects' actual keypress 
responses were spatially compatible with the side of space on which the imperative signal was presented. In contrast, when making the pitch judgments, there was no spatial correspondence between any aspect of the stimulus and the side of response. As Simon (1990) has documented, when stimuli and their respective responses are spatially compatible, performance may well be facilitated, because of a tendency to react toward the location of the stimulus source. As a consequence, the contrast between the different stimulus-response compatibilities across the pitch and position tasks used here also suggests that subjects will be impaired in responding on the basis of pitch, as compared with responding on the basis of position.

In addition to the expectation that RTs to the position task would be faster than RTs to the pitch task, it was also expected, on the basis of the previous work, that response repetition effects should be evident for the two tasks. Finally, on the grounds that the pitch and position judgments demanded the invocation of different chains of underlying processes (Rogers \& Monsell, 1995), it was predicted that there should be an overall cost in switching tasks across trials, as compared with the case in which no such switch occurred.

\section{METHOD}

\begin{abstract}
Apparatus
Visual information was presented on a 14-in. Acorn color monitor. The monitor was at a fixed distance from a chinrest. When a subject sat at the chinrest, the viewing distance from the screen was $57 \mathrm{~cm}$. The chinrest was adjusted for each subject so that the center of the screen was at eye level. The timing, stimulus presentation, and data collection were under the control of an Acorn Archimedes R260 computer. Two finger response keys were provided and interfaced with the computer. Tones were presented via a pair of Sennheiser HD 56 earphones. All testing was carried out in a small testing room, and all of the equipment, except for the computer, was housed in this room.
\end{abstract}

\section{Stimuli and Design}

To maintain parity with previous studies reported by Quinlan and Hill (in press), a $3 \times 3$ array of small square boxes was presented on each trial. Each box measured approximately $1^{\circ} \times 1^{\circ}$ of visual angle. Owing to screen distortion, the horizontal center-tocenter distance of the boxes was $5.0^{\circ}$ of visual angle, but the vertical center-to-center distance was $4.5^{\circ}$ of visual angle. All of the subjects were run with the lights off in a small testing room. Under these conditions, the background luminance of the VDU screen was $0.11 \mathrm{~cd} / \mathrm{m}^{2}$. The luminance of the contour of an individual box was $0.50 \mathrm{~cd} / \mathrm{m}^{2}$.

The auditory stimuli were pure tones. The tones were produced digitally (8-bit quantization) with a sampling rate of $41.7 \mathrm{kHz}$. Because of the rather awkward manner in which the Archimedes presents and generates tones, tight constraints were placed on producing the set of tone stimuli. Within this system, values of pitch and duration covary; hence, the relative values of tone frequency were not ideal. On each trial, a reference signal was presented prior to the presentation of the imperative signal. The reference signal was a $488-\mathrm{Hz}$ binaural tone and was identical to the auditory preparatory signal used by Quinlan and Hill (in press). In the cases in which a high-pitch tone was used as the target, its frequency was $500 \mathrm{~Hz}$, and in the cases in which a low-pitch tone was used as the target, its frequency was $465 \mathrm{~Hz}$. These particular frequencies were chosen because both of the targets could be presented for $150 \mathrm{msec}$. The reference signal frequency was set at $488 \mathrm{~Hz}$, because this frequency could be presented for 50 and $150 \mathrm{msec}$. The stimulus for the positional judgment was a $488-\mathrm{Hz}$ tone presented monaurally for $150 \mathrm{msec}$. Given the particular hardware constraints, it meant that the reference signal was slightly above the midpoint between the designated high and the designated low target frequencies. Stimulus intensities gave peak sound pressure levels of $86 \mathrm{~dB}(\mathrm{~A})$ at the headphones, calibrated with a Bruel and Kjaer artificial ear and sound level meter. The same reference signal was used on all the trials in the experiment.

In generating the experimental trials, the starting point was to set out a pair of trials. The first member of each pair $(\operatorname{trial} n)$ was known as the prime trial, and the second member of each pair $(\operatorname{trial} n+1)$ was known as the target trial. Each trial contained a reference signal followed by an imperative signal. For ease of exposition, the imperative signal on the prime trial will be referred to as the prime stimulus, and the imperative signal on the target trial will be referred to as the target stimulus. In each experiment, 16 basic pairs of trials were generated. This set of 16 corresponded to the factorial combinations of prime judgment (pitch vs. position), target judgment (pitch vs. position), response type (same as prime vs. different from prime), and stimulus pair (high/low vs. left/right). Random orders of these 16 pairs of trials gave rise to the set of trials used in the individual experiments.

For the pitch task (henceforth, on the pitch trials), the binaural reference signal was followed by another binaural tone of a higher pitch (the high tone) or by a binaural tone of a lower pitch (the low tone). The right-hand response key was designated the high key, and the left-hand key was designated the low key. For the position task (henceforth, on the position trials), the binaural tone cue was followed by a monaural tone presented to the right ear (the right tone) or by a monaural tone to the left ear (the left tone). The righthand response key was designated the right key, and the left-hand key was designated the left key. In using this particular mapping, compatible stimuli were assigned to the same actual keypress (see Campbell \& Proctor, 1993; Mudd, 1963), although, as has been noted, the strict spatial compatibility only existed for responses on the positional judgment trials.

Although the reference signals in this experiment may seem redundant, it was felt important that the subjects need not attempt to remember the particular pitches of the high and low tones. The pitch of the target could be judged relative to the pitch of the reference. The subjects could therefore base their response on the detection of a falling or a rising pitch. To maintain parity with the pitch trials, the same reference signal used on the pitch trials was presented on the position trials.

\section{Subjects}

Twelve subjects selected from the Department of Psychology subject panel participated in the experiment. Psychology student subjects received course credit for participation. Other students were paid $£ 3 /$ session. All reported having normal or corrected-tonormal vision, and none reported any known hearing problems.

\section{Procedure}

Each subject was tested individually. Prior to the experiment, the subjects were given verbal instructions about the task constraints. The nature of each trial was explained in detail. On each trial, the sequence of events was as follows. Initially, a central fixation plus sign was presented in the center box for $500 \mathrm{msec}$. The plus was $0.3^{\circ} \times 0.4^{\circ}$ of visual angle (luminance $=2.00 \mathrm{~cd} / \mathrm{m}^{2}$ ). The fixation plus was immediately replaced by the reference signal for $50 \mathrm{msec}$. The imperative target tone was presented at the offset of the cue for $150 \mathrm{msec}$, and the subjects had up to $3,000 \mathrm{msec}$ to respond. When 
(i) (ii) (iii) (iv) (v) (vi) (i) (ii) (iii)
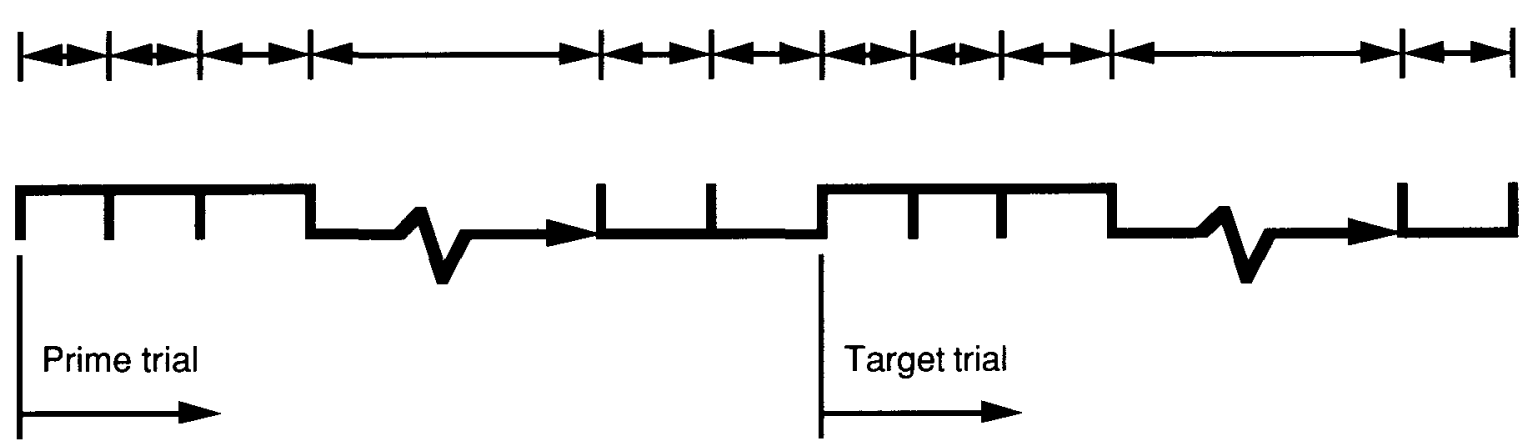

Time (not to scale)

(i) Fixation cross (500 msec)
(ii) Reference signal (50 msec)
(iii) Imperative signal (150 msec)
(iv) RT
(v) Visual feedback (see legend)
(vi) ITI (500 msec)

Figure 1. Schematic representation of the sequence of events on a pair of prime and target trials. Following a correct response, the visual feedback was left on for $500 \mathrm{msec}$, and after an incorrect response, an additional 400 msec was added to this period.

the computer detected a response or the response deadline expired, contingent feedback was presented on the screen. The word Correct or Error was presented slightly below the position of the overall array of boxes. This lasted for $500 \mathrm{msec}$, and if a mistake had been detected, an additional $400 \mathrm{msec}$ was added to this interval. Next, the screen went blank for $500 \mathrm{msec}$. After a correct response, therefore, the intertrial interval was set at $1 \mathrm{sec}$. Figure 1 provides a schematic representation of the sequence of events on a pair of prime and target trials.

Following the verbal instructions, the subjects were told that responses would be timed and that they should try to respond quickly without making too many errors. The experimenter then left the room, and the subject worked through a single block of 32 practice trials. This block of trials contained a random order of the basic 16 pairs of trials. Following this practice block, the subjects were told of the overall task demands and were asked to sign a consent form They were then informed that the experimental trials were divided into five further blocks and that a message would be presented on the screen when a block had finished. To initiate the next block, they were instructed to press a foot switch and to defer this if they wanted to rest. Although the subjects were not so informed, each experimental block of trials contained 128 individual trials. Each experimental block contained eight random orders of the 16 basic pairs of trials. Across the five blocks of trials, the subjects were presented with 640 experimental trials in total - that is, 320 prime- target pairs. Overall, a given testing session lasted approximately $40 \mathrm{~min}$.

\section{RESULTS}

Throughout the experimental trials, every odd-numbered trial was a prime trial, and every even trial was a target trial (as is shown in Figure 1). Initially, mean RTs were computed only over target trials, and in order for a trial's score to be included, the response on both the prime trial and the target trial needed to have been correct. That is, only the data for even-numbered trials were analyzed. Responses were averaged over the actual keypress made (i.e., left keypress vs. right keypress), and Table 1 shows the summary statistics of the data that were analyzed. There were no target trials on which any subject failed to respond. The total number of errors per condition for each subject were noted, and the corresponding mean error rates are included in Table 1.

The RT data from the target trials were entered into a $2 \times 2 \times 2$ repeated measures analysis of variance. Here, target judgment (pitch vs. position), task repetition (pres- 
Table 1

Summary Statistics for Performance on the Target Trials in Experiment 1

\begin{tabular}{|c|c|c|c|c|}
\hline \multirow[b]{4}{*}{ Response Repetition } & \multicolumn{4}{|c|}{ Target Judgment } \\
\hline & \multicolumn{2}{|c|}{ Position } & \multicolumn{2}{|c|}{ Pitch } \\
\hline & \multicolumn{4}{|c|}{ Task Repetition } \\
\hline & $\begin{array}{c}\text { Present } \\
\text { (Position) }\end{array}$ & $\begin{array}{l}\text { Absent } \\
\text { (Pitch) }\end{array}$ & $\begin{array}{l}\text { Present } \\
\text { (Pitch) }\end{array}$ & $\begin{array}{c}\text { Absent } \\
\text { (Position) }\end{array}$ \\
\hline \multicolumn{5}{|l|}{ Present } \\
\hline Mean RT $(S D)$ & $376(53)$ & $441(64)$ & $415(94)$ & $522(163)$ \\
\hline$\%$ Errors & 2.7 & 5.8 & 3.3 & 9.6 \\
\hline \multicolumn{5}{|l|}{ Absent } \\
\hline Mean RT (SD) & $383(56)$ & $426(91)$ & $457(104)$ & $483(146)$ \\
\hline$\%$ Errors & 2.2 & 2.5 & 3.5 & 7.1 \\
\hline
\end{tabular}

ent vs. absent), and response repetition (present vs. absent) were entered as fixed factors. Subjects acted as a random factor. This analysis revealed statistically significant main effects of target judgment $[F(1,11)=5.69, p<$ $.05]$ and task repetition $[F(1,11)=17.53, p<.01$; $F(1,11)=0.03, p>.05$, for the main effect of response repetition]. The only interaction to reach statistical significance was the task repetition $\times$ response repetition interaction $[F(1,11)=26.42, p<.001 ; F(1,11)=0.45, p>$ .05 , for the task judgment $\times$ task repetition interaction; $F(1,11)=0.143, p>.05$, for the task repetition $\times$ response repetition interaction; and $F(1,11)=2.63, p>.05$, for the target judgment $\times$ task repetition $\times$ response repetition interaction].

The main effect of target judgment is easy to interpret, given the lack of any statistically significant interactions with this factor. As was predicted, the data showed that RTs were faster to the position judgment than to the pitch judgment. The overall mean RT on the position trials was $407 \mathrm{msec}$, and it was $470 \mathrm{msec}$ on the pitch trials.

The task repetition $\times$ response repetition interaction was further decomposed, using a Tukey's HSD test. This revealed that when the same judgment was performed on the prime and target trials, RTs were quicker when the response was the same on both prime and target trials than when the prime and target trials demanded different responses $(p<.05)$. In contrast, when different judgments were performed on the prime and target trials, RTs were quicker when the response was different across these trials than when the same response was repeated $(p<.05)$. Furthermore, RTs were generally quicker when the same judgment was repeated over the trials than when different judgments were used on the prime and target trials [ $p<$ .05 , all comparisons; critical range $(\alpha=.05)=23$ ].

Error scores were arcsine transformed prior to analysis (see Winer, 1962). These transformed scores were analyzed in the same manner as the RTs. This analysis revealed statistically significant main effects of both task repetition $[F(1,11)=15.25, p<.05]$ and response repetition $[F(1,11)=5.42, p<.05 ; F(1,11)=2.65, p>.05$, for the main effect of target judgment]. Although the task repetition $\times$ response repetition interaction approached statistical significance $[F(1,11)=4.50, p=.058]$, the only interaction to reach statistical significance was that between target judgment and task repetition $[F(1,11)=$ $6.52, p<.5 ; F(1,11)=0.62, p>.05$, for the target judgment $\times$ task repetition interaction; $F(1,11)=0.51, p>$ .05 , for the target judgment $\times$ task repetition $\times$ response repetition interaction].

Further analysis of the target judgment $\times$ task repetition interaction revealed that the subjects were particularly inaccurate when making a pitch judgment immediately after a position judgment $[p<.05$, all comparisons, using the HSD test; critical range $(\alpha=.05)=0.1180]$. The transformed mean values entered into the test were 0.2041 , $0.2727,0.3220$, and 0.5234 , respectively, for the trials on which a position judgment was repeated, the trials on which a pitch judgment was repeated, the trials on which a task switch occurred prior to a position judgment, and the trials on which a task switch occurred prior to a pitch judgment. This particular result may well reflect the difficulty the subjects had in performing the pitch task. If the subjects attempted to remember the particular highand low-pitch values over trials, the chance of forgetting these was enhanced when performing the pitch task immediately after the position task. Under these conditions and within a block of trials, there was always at least one position trial interleaved between performing two pitch trials. On these grounds, the effect in the accuracy data may reflect a problem in forgetting either the nature of the pitch discrimination or the appropriate response mapping for this task. Generally speaking, however, the analysis of the RTs and errors are in agreement, and there was no evidence of any speed/error tradeoffs in the data.

\section{GENERAL DISCUSSION}

The results of the experiment were in agreement with the predictions set out initially. First, the subjects produced faster RTs in the position than in the pitch task. Such a result was predicted both on the basis of the relative difference in task difficulty across the pitch and position judgments and also because of the difference in the stimulus-response compatibilities used in the two tasks. Clearly, further work might examine the consequences of equating the difficulty of the pitch and the position judgments. Second, both repetition and task-switching effects were revealed in the data. Although a standard repetition effect was present in the data, clear differences in performance were present, depending on whether or not subjects carried out the same task on prime and target trials. When the same task was performed on prime and target trials, there was a strong response repetition effect. The subjects were quicker to respond when the prime and target trials demanded the same response than when they demanded different responses. When the task was different on the prime and target trials, completely the reverse effect obtained: The subjects were now quicker to make different responses on prime and target trials than they 
were to repeat the same response. Moreover, the subjects were generally slower in responding following a task change than when there was no task change.

Initially, interest is with the overall pattern of effects that arose on both the pitch and the position trials. The subjects were facilitated in repeating the same response if the same task was performed on the prime and the target trials, and they were impeded in repeating the same response if there was a task switch between the prime and the target trials. The most straightforward account of this pattern of performance is that provided by the bypass rule (Fletcher \& Rabbitt, 1978; Krueger \& Shapiro, 1981). According to this account, the rule describes two tendencies - namely, "(i) to repeat the response if the stimulus was repeated from the preceding trial, but (ii) change the response if the stimulus was changed" (Krueger \& Shapiro, 1981, p. 242). The first of these tendencies describes well the finding that the subjects were facilitated when both the response and the stimulus were repeated over the prime and target trials. Indeed, the present result shows, yet again, that if the stimulus and the response are repeated over adjacent trials, RTs will be facilitated. Such a finding accords well with the short-cut hypothesis put forward by Pashler and Baylis (1991). They argued that this type of repetition effect was due to the formation of "shortcuts to response selection" (p. 43), with a particular stimulus directly invoking the appropriate response when it is repeated.

The second of the tendencies described by the bypass rule also provides something of an explanation as to why the subjects were impeded when the prime and the target stimuli differed but the same keypress had to be repeated. Given that the difference in the stimuli had been registered, the tendency to respond different in some sense interfered with the process of having to repeat the same response. Although such an account appears to be relatively straightforward, it does depend on accepting various assumptions about the nature of the underlying mechanisms that are not explicitly stated in the rule. For instance, in order to account for the poor performance on sameresponse trials following a task switch, it must be assumed that the imperative signal on a given trial was classified as belonging to a particular response category. The slowing on same-response trials following a task switch cannot simply be due to some impediment in repeating the response, having merely detected a pitch difference between the prime and the target stimuli. Consider the case in which the prime stimulus was a low-pitch tone and the target stimulus was a high-pitch tone. Here, the response differed between the prime and the target trials, but RTs were never as slow as when, say, a low-pitch prime stimulus preceded a left monaural target stimulus. In this latter case, the responses were the same across the prime and target trials, but there was also a pitch difference between the stimuli across the two trials. Merely detecting a pitch difference between the prime and the target stimuli cannot account for the overall slower responding when the same response was needed after a task switch. It is true that whenever the prime and target stimuli differed, there was a slowing on target trials, but an additional finding was that RTs were particularly slow when both the stimuli and the task differed across the prime and target trials. This suggests that not only was the difference between the prime and the target detected, but also that both had also been assigned to the same response category. On these grounds, it appears that the subjects were particularly impeded when type-distinct stimuli were grouped into the same response category. Indeed, Pashler and Baylis (1991) and Smith (1968) have also reported the finding that responses are particularly impeded when the response, but not the stimulus, was the same and when "there was no obvious conceptual grouping of the stimuli assigned to given responses" (Pashler \& Baylis, 1991, p. 38).

Although some might argue that the bypass rule provides a complete account of the data, it is of some interest to note the presence of task-switching effects (Rogers \& Monsell, 1995). The data show that the subjects were generally slower to execute a response when there was a task switch between the prime and the target trials than when there was no such switch. Such a task-switching effect does imply that the two different tasks invoked distinct underlying processes and that switching between them carried a time penalty. Indeed, the presence of this effect seems to suggest that, in addition to the same-different matching processes implicated by the bypass rule, the experiment has revealed costs associated with task-set reconfiguration (Rogers \& Monsell, 1995). At a very simple level, the positional judgment could have been based on the detection of peripheral stimulation at a given ear--for instance, a discernible pressure change at the eardrum. In contrast, the earliest place at which pitch information could have been recovered is at the cochlea (Moore, 1997). One possibility, therefore, is that the cost of a task switch arose because the position and pitch judgments depended on read-off of information from different levels of the auditory system. In this sense, the two tasks demanded different underlying processes, and switching between these different processes resulted in a contingent time penalty.

In summary, the present study has revealed that performance on even very basic auditory judgments can be affected by immediately prior events. In line with predictions from the literature on visual judgments, it is clear that performance in auditory CRTs on the present trial is facilitated if both the stimulus and the response are repeated. Moreover, any change in the nature of the stimulus from the previous trial can lead to a time penalty on the present trial. In these respects, performance in the reported auditory CRTs conforms to the basic tendencies described by the bypass rule. However, the subjects were also generally impaired following a task switch, as compared with cases in which the same task was repeated, and particular difficulties arose when different members of the same artificial category occurred sequentially. Overall, therefore, this study seems to suggest that when successive stimuli are presented within the same sensory modality and each demands a different judgment, general process- 
ing constraints apply. Such constraints are well described by the bypass rule. Nevertheless, particular and additional costs may also arise ( 1 ) because the different tasks invoke quite different chains of processes, and (2) when the stimuli are classified into artificial categories.

\section{REFERENCES}

Allport, A., Styles, E. A., \& Hsieh, S. (1994). Shifting intentional set: Exploring the dynamic control of tasks. In C. Umiltà \& M. Moscovitch (Eds.), Attention and performance, XV: Conscious and nonconscious information processing (pp. 421-452). Cambridge, MA: MIT Press.

Ben-Artzi, E., \& MARKs, L. E. (1995). Visual-auditory interaction in speeded classification: Role of stimulus difference. Perception \& Psychophysics, 57, 1151-1162.

Bertelson, P. (1965). Serial choice reaction-time as a function of response versus signal-and-response repetition. Nature, 206, 217-218.

Campbell, K. C., \& Proctor, R. W. (1993). Repetition effects with categorizable stimulus and response sets. Journal of Experimental Psychology: Learning, Memory, \& Cognition, 19, 1345-1362.

FletCher, B., \& RABBitT, P. M. A. (1978). The changing pattern of perceptual analytic strategies and response selection with practice in a two-choice reaction time task. Quarterly Journal of Experimental Psychology, 30, 417-427.

Krueger, L. E., \& SHAPIRO, R. G. (1981). Intertrial effects of samedifferent judgements. Quarterly Journal of Experimental Psychology, 33A, 241-265.

Moore, B. C. J. (1997). An introduction to the psychology of hearing (4th ed.). San Diego: Academic Press.

MudD, S. A. (1963). Spatial stereotypes of four dimensions of pure tone. Journal of Experimental Psychology, 66, 347-352.

Pashler, H., \& Baylis, G. (1991). Procedural learning: 2. Intertrial repetition effects in speeded-choice judgments. Journal of Experimental Psychology: Learning, Memory, \& Cognition, 17, 33-48.
PosNer, M. I., \& CoHEN, Y. (1984). Components of visual orienting. In H. Bouma \& D. G. Bouwhuis (Eds.), Attention and performance X: Control of language processes (pp. 531-556). Hillsdale, NJ: Erlbaum

Quinlan, P. T., \& HILl, N. I. (in press). Sequential effects in rudimentary auditory and visual tasks. Perception \& Psychophysics.

RABBITT, P. M. A. (1992). Many happy repetitions: A celebration of the "Bertelson Repetition Effect" 1961-1991. In J. Alegria, D. Holender, J. Junca, \& M. Radeau (Eds.), Analytic approaches to human cognition (pp. 313-330). Amsterdam: Elsevier.

Reuter-Lorenz, P. A., Jha, A. P., \& Rosenquist, J. N. (1996). What is inhibited in inhibition of return? Journal of Experimental Psychology: Human Perception \& Performance, 22, 367-378.

RoGers, R. D., \& MONSEll, S. (1995). Costs of a predictable switch between simple cognitive tasks. Journal of Experimental Psychology. General, 124, 207-231.

SCHARF, B. (1998). Auditory attention: The psychoacoustical approach. In H. Pashler (Ed.), Attention (pp. 75-117). Hove, U.K.: Psychology Press.

Simon, J. R. (1990). The effects of an irrelevant directional cue on human information processing. In R. W. Proctor \& T. G. Reeve (Eds.), Stimulus-response compatibility (pp. 31-86). Amsterdam: Elsevier, North-Holland.

SMIтH, M. C. (1968). Repetition effect and short-term memory. Journal of Experimental Psychology, 77, 435-439.

SPENCE, C., \& Driver, J. (1997). On measuring selective attention to an expected modality. Perception \& Psychophysics, 59, 389-403.

Terry, K. M., Valdes, L. A., \& Neill, W. T. (1994). Does “inhibition of return" occur in discrimination tasks? Perception \& Psychophysics, 55, 279-286.

WINER, B. J. (1962). Statistical principles in experimental design. New York: McGraw-Hill.

(Manuscript received August 19, 1997; revision accepted for publication April 13, 1998.) 